PROCEEDINGS OF THE

AMERICAN MATHEMATICAL SOCIETY

Volume 129, Number 9, Pages 2655-2662

S 0002-9939(01)06050-6

Article electronically published on February 9, 2001

\title{
ON THE INTERSECTION OF TWO-PARAMETER MEAN VALUE FAMILIES
}

\author{
HORST ALZER AND STEPHAN RUSCHEWEYH
}

(Communicated by David Preiss)

Abstract. We determine all means which are in the intersection of two multivariable two-parameter mean value families. These families were introduced by C. Gini (1938) and K.B. Stolarsky (1975).

\section{INTRODUCTION AND MAIN RESULT}

In 1938, C. Gini [4] presented the following two-parameter mean value family of $n$ variables. Let $u$ and $v$ be real numbers and let $\mathbf{x}=\left(x_{1}, \ldots, x_{n}\right)$ be an $n$-tuple of positive real numbers. Then we define

$$
G(u, v ; \mathbf{x})=\left[\sum_{k=1}^{n} x_{k}^{u} / \sum_{k=1}^{n} x_{k}^{v}\right]^{1 /(u-v)} \quad(u \neq v)
$$

and

$$
G(u, u ; \mathbf{x})=\lim _{v \rightarrow u} G(u, v ; \mathbf{x})=\exp \left(\sum_{k=1}^{n} x_{k}^{u} \log x_{k} / \sum_{k=1}^{n} x_{k}^{u}\right) .
$$

This family includes the well-known power means

$$
G(u, 0 ; \mathbf{x})=\left(\frac{1}{n} \sum_{k=1}^{n} x_{k}^{u}\right)^{1 / u} \quad(u \neq 0), \quad G(0,0 ; \mathbf{x})=\prod_{k=1}^{n} x_{k}^{1 / n} .
$$

Besides the geometric mean $G(0,0 ; \mathbf{x})$, the two other classical mean values are special power means: the arithmetic mean

$$
G(1,0 ; \mathbf{x})=\frac{1}{n} \sum_{k=1}^{n} x_{k}
$$

and the harmonic mean

$$
G(-1,0 ; \mathbf{x})=\left(\frac{1}{n} \sum_{k=1}^{n} \frac{1}{x_{k}}\right)^{-1}
$$

The properties of these means have been discussed intensively by many authors. We refer to [2, 3, 6, 12, 14, 16] and the references therein.

Received by the editors January 10, 2000.

2000 Mathematics Subject Classification. Primary 26B99; Secondary 30B10, 30B40, 30D30, $33 \mathrm{C} 05$.

Key words and phrases. Gini means, Stolarsky means, hypergeometric function. 
In 1975, K.B. Stolarsky 19 introduced a new two-parameter family of multivariable mean values, which E.B. Leach \& M.C. Sholander referred to as "the most interesting mean value formula that we have seen" [9, p. 222]. It is built from the quotient of divided differences (initially taken from $n$ distinct positive numbers $\left.x_{1}, \ldots, x_{n}\right)$ of the functions $x^{r}$ and $x^{s}$, with distinct real numbers $r$ and $s$ :

$$
\sum_{k=1}^{n} \frac{x_{k}^{r}}{Q^{\prime}\left(x_{k}\right)}, \quad \sum_{k=1}^{n} \frac{x_{k}^{s}}{Q^{\prime}\left(x_{k}\right)}, \quad\left(Q(x)=\prod_{k=1}^{n}\left(x-x_{k}\right)\right),
$$

normalized by proper factors and exponents, in order to make this expression a "mean". This leads to

$$
E(r, s ; \mathbf{x})=\left[\frac{(s-n+2)_{n-1} \sum_{k=1}^{n} x_{k}^{r} / Q^{\prime}\left(x_{k}\right)}{(r-n+2)_{n-1} \sum_{k=1}^{n} x_{k}^{s} / Q^{\prime}\left(x_{k}\right)}\right]^{1 /(r-s)},
$$

where $(a)_{b}=\Gamma(a+b) / \Gamma(a)$ denotes the Pochhammer symbol. This expression makes sense also for the limiting cases where some or all of the $x_{k}$ are equal, and also, taking limits again, for all choices of real $r, s$. The arithmetic, geometric, and harmonic means are the special cases $(r, s)=(n, n-1),(n-1,-1),(-1,-2)$, respectively.

The definition (1.2) yields for $n=2$ :

$$
E\left(r, s ; x_{1}, x_{2}\right)=\left[\frac{s}{r} \frac{x_{1}^{r}-x_{2}^{r}}{x_{1}^{s}-x_{2}^{s}}\right]^{1 /(r-s)} .
$$

This family contains the well-known logarithmic mean

$$
E\left(1,0 ; x_{1}, x_{2}\right)=\frac{x_{1}-x_{2}}{\log x_{1}-\log x_{2}}
$$

and the identric mean

$$
E\left(1,1 ; x_{1}, x_{2}\right)=\frac{1}{e}\left(x_{1}^{x_{1}} / x_{2}^{x_{2}}\right)^{1 /\left(x_{1}-x_{2}\right)},
$$

which have been studied thoroughly in the recent past. In particular, there exist many interesting inequalities for both means in the literature. The logarithmic mean has remarkable applications in mathematical physics, chemistry, meteorology, and economics; see [7, 9, 17, 18. The identric mean plays a central role in $E$ because of the known integral representation

$$
E\left(r, s ; x_{1}, x_{2}\right)=\exp \left(\frac{1}{r-s} \int_{s}^{r} \frac{1}{t} \log E\left(1,1 ; x_{1}^{t}, x_{2}^{t}\right) d t\right),
$$

which shows that $E$ can be expressed in terms of the identric mean. Monotonicity and comparison theorems as well as further properties of the family (1.3) and its multivariable generalization (1.2) are given in [8, 9, 10, 13, 15, 16.

H.W. Gould \& M.E. Mays pointed out a "strong analogy" [5, p. 400] between $G$ and $E$. Indeed, it has been proved that both families have similar properties. For instance, $G(u, v ; \mathbf{x})$ and $E(r, s ; \mathbf{x})$ are increasing with respect to their parameters.

Our paper has been inspired by two articles published by D.H. Lehmer [11] in 1971 and by H.W. Gould \& M.E. Mays [5] in 1984. These authors investigated the intersection of certain mean value families of two variables. Lehmer proved that the only means which belong to $G\left(u, u-1 ; x_{1}, x_{2}\right)$ and $E\left(r, 2 r ; x_{1}, x_{2}\right)$ are the arithmetic, geometric, and harmonic means. We note that the main tool in 
Lehmer's proof are the Maclaurin series expansions of $t \mapsto G(u, u-1 ; 1,1-t)$ and $t \mapsto E(r, 2 r ; 1,1-t)$. The same method of proof was used by Gould \& Mays to establish that $G\left(u, u-1 ; x_{1}, x_{2}\right)$ and $E\left(r, s ; x_{1}, x_{2}\right)$ also have in common only the three classical means.

It is natural to look for an extension of these results, namely to characterize those means which belong to both, $G(u, v ; \mathbf{x})$ and $E(r, s ; \mathbf{x})$, where $n \geq 2$ is a fixed integer. It is the aim of this paper to solve this problem. In contrast with the previous approaches we make use of some simple facts from analytic function theory, which are employed to provide a crucial relation between the parameters involved. A basic observation in our proof is formula (2.1) below, which links $E$ with the classical Gaussian hypergeometric function

$$
{ }_{2} F_{1}(a, b ; c ; z)=\sum_{k=0}^{\infty} \frac{(a)_{k}(b)_{k}}{(c)_{k}} \frac{z^{k}}{k !} \quad(|z|<1),
$$

whose properties and representations are decisive for the multivariable case. A detailed collection of the basic facts on hypergeometric functions can be found, for instance, in [1] chapter 2].

Our main result is as follows.

Theorem. The only means which belong to both $G(u, v ; \mathbf{x})$ and $E(r, s ; \mathbf{x})$ are

(i) the power means, if $n=2$, and

(ii) the arithmetic, geometric, and harmonic means, if $n \geq 3$.

The Theorem implies the following result, which has been conjectured by E.B. Leach \& M.C. Sholander [9].

Corollary. For $n \geq 3$, the arithmetic, geometric, and harmonic means are the only power means which belong to $E(r, s ; \mathbf{x})$.

The proof of the Theorem, which is given in the following sections, would have been much more cumbersome without the availability of computer algebra. We used Mathematica 4.0 for series expansions and the solution of systems of algebraic equations. However, the more delicate parts of those calculations have been verified independently, using "paper and pencil".

\section{A LEMMA}

The following lemma plays an important role in the proof of our Theorem.

Lemma. For $n \geq 2,|y-1|<1$, we have

$$
[E(r, s ; y, 1, \ldots, 1)]^{r-s}=\frac{{ }_{2} F_{1}(1, n-1-r ; n ; 1-y)}{{ }_{2} F_{1}(1, n-1-s ; n ; 1-y)} .
$$

Proof. Let $y \neq 1$ be a positive number and let $Q$ be as in (1.1). Setting $x_{1}=y$ and $x_{j}=1+j \lambda(j=2, \ldots, n)$, we get for $\lambda \rightarrow 0$ :

$$
\sum_{k=1}^{n} \frac{x_{k}^{r}}{Q^{\prime}\left(x_{k}\right)}=\frac{y^{r}}{(y-1)^{n-1}}+S(r, n, y ; \lambda)+o(1)
$$

where

$$
S=S(r, n, y ; \lambda)=\lambda^{2-n} \sum_{k=2}^{n}(-1)^{n-k} \frac{(1+k \lambda)^{r}}{(k-2) !(n-k) !(1-y+k \lambda)} .
$$


We obtain

$$
\begin{aligned}
(n-2) & !(1-y) \lambda^{n-2} S \\
= & \sum_{k=2}^{n}(-1)^{n-k}\left(\begin{array}{l}
n-2 \\
k-2
\end{array}\right) \frac{1-y}{1-y+k \lambda}(1+k \lambda)^{r} \\
= & \sum_{k=2}^{n}(-1)^{n-k}\left(\begin{array}{l}
n-2 \\
k-2
\end{array}\right) \frac{1-y}{1-y+k \lambda} \sum_{m=0}^{\infty}\left(\begin{array}{c}
r \\
m
\end{array}\right)(k \lambda)^{m} \\
= & \sum_{k=2}^{n}(-1)^{n-k}\left(\begin{array}{l}
n-2 \\
k-2
\end{array}\right) \sum_{j=0}^{\infty}\left(\frac{-k \lambda}{1-y}\right)^{j} \sum_{m=0}^{\infty}\left(\begin{array}{c}
r \\
m
\end{array}\right)(k \lambda)^{m} \\
= & \sum_{k=2}^{n}(-1)^{n-k}\left(\begin{array}{l}
n-2 \\
k-2
\end{array}\right) \sum_{j=0}^{\infty}\left(\frac{-k \lambda}{1-y}\right)^{j} \sum_{m=j}^{\infty}\left(\begin{array}{c}
r \\
m-j
\end{array}\right)(k \lambda)^{m-j} \\
= & \sum_{k=2}^{n}(-1)^{n-k}\left(\begin{array}{l}
n-2 \\
k-2
\end{array}\right) \sum_{m=0}^{\infty} \sum_{j=0}^{m}\left(\begin{array}{c}
r \\
m-j
\end{array}\right)\left(\frac{-k \lambda}{1-y}\right)^{j}(k \lambda)^{m-j} \\
= & \sum_{m=0}^{\infty} \sum_{j=0}^{m}\left(\begin{array}{c}
r \\
m-j
\end{array}\right)(y-1)^{-j}\left[\sum_{k=2}^{n}(-1)^{n-k}\left(\begin{array}{l}
n-2 \\
k-2
\end{array}\right) k^{m}\right] \lambda^{m} .
\end{aligned}
$$

Applying the formula

$$
\sum_{k=2}^{n}(-1)^{n-k}\left(\begin{array}{l}
n-2 \\
k-2
\end{array}\right) k^{m}= \begin{cases}0, & \text { if } m \in\{0, \ldots, n-3\} \\
(n-2) !, & \text { if } m=n-2,\end{cases}
$$

which can be proved easily by induction on $n$, we get

$$
S=-\sum_{j=0}^{n-2}\left(\begin{array}{c}
r \\
n-2-j
\end{array}\right)(y-1)^{-j-1}+o(1) .
$$

Hence, (2.2) and (2.3) yield

$$
\sum_{k=1}^{n} \frac{x_{k}^{r}}{Q^{\prime}\left(x_{k}\right)}=(y-1)^{1-n}\left[y^{r}-\sum_{j=0}^{n-2}\left(\begin{array}{l}
r \\
j
\end{array}\right)(y-1)^{j}\right]+o(1) \quad(\lambda \rightarrow 0) .
$$

From (1.2) and (2.4), and assuming that

$$
r, s \notin\{0,1, \ldots, n-2\},
$$

we obtain

$$
[E(r, s ; y, 1, \ldots, 1)]^{r-s}=\frac{(s-n+2)_{n-1}\left[y^{r}-\sum_{j=0}^{n-2}\left(\begin{array}{l}
r \\
j
\end{array}\right)(y-1)^{j}\right]}{(r-n+2)_{n-1}\left[y^{s}-\sum_{j=0}^{n-2}\left(\begin{array}{c}
s \\
j
\end{array}\right)(y-1)^{j}\right]} .
$$

Using

$$
\left(\begin{array}{c}
r \\
j
\end{array}\right)=\left(\begin{array}{c}
r \\
n-1
\end{array}\right)(-1)^{k} \frac{(1)_{k}(n-1-r)_{k}}{(n)_{k} k !} \quad \text { with } \quad k=j-n+1,
$$


we get for $|y-1|<1$ :

$$
\begin{aligned}
& y^{r}-\sum_{j=0}^{n-2}\left(\begin{array}{l}
r \\
j
\end{array}\right)(y-1)^{j}=\sum_{j=n-1}^{\infty}\left(\begin{array}{l}
r \\
j
\end{array}\right)(y-1)^{j} \\
= & \left(\begin{array}{c}
r \\
n-1
\end{array}\right) \sum_{k=0}^{\infty}(-1)^{k} \frac{(1)_{k}(n-1-r)_{k}}{(n)_{k} k !}(y-1)^{k+n-1} \\
= & \frac{(r-n+2)_{n-1}}{(n-1) !}(y-1)^{n-1}{ }_{2} F_{1}(1, n-1-r ; n ; 1-y) .
\end{aligned}
$$

From (2.6) and (2.7) we obtain (2.1), where we obviously can eliminate the restriction (2.5).

Remarks. (1) The hypergeometric function can be continued analytically from the unit disc along any curve which does not pass through the point 1 . Hence, (2.1) implies that

$$
f_{r, s}(z)=\left[E\left(r, s ; e^{z}, 1, \ldots, 1\right)\right]^{r-s}
$$

is meromorphic in $\mathbf{C}$.

(2) It is well known that $w(x)={ }_{2} F_{1}(a, b ; c ; x)$ satisfies the following linear homogeneous differential equation of second order:

$$
D_{w}(x)=w^{\prime \prime}(x)+\frac{c-(a+b+1) x}{x(1-x)} w^{\prime}(x)-\frac{a b}{x(1-x)} w(x) \equiv 0
$$

(see [1, p. 75]). Let $x_{0} \neq 0,1$, and assume $w\left(x_{0}\right)=w^{\prime}\left(x_{0}\right)=0$. Then from the relations $D_{w}^{(k)}\left(x_{0}\right)=0, k=0,1, \ldots$, we get, by induction, $w^{(k)}\left(x_{0}\right)=0, k=0,1, \ldots$, which would imply $w \equiv 0$, contradicting $w(0)=1$. Thus, $z \mapsto{ }_{2} F_{1}\left(a, b ; c ; 1-e^{z}\right)$ has in $\mathbf{C}$ at most simple zeros. From (2.1) and (2.8) we conclude that all poles and zeros of $f_{r, s}$ (with $r \neq s$ ) are simple.

(3) Euler's integral representation

$$
{ }_{2} F_{1}(1, n-r-1 ; n ; x)=(n-1) \int_{0}^{1}(1-t)^{n-2} \frac{d t}{(1-t x)^{n-r-1}}
$$

(see [1, p. 65]) implies that

$$
x \mapsto \frac{\partial}{\partial r}{ }_{2} F_{1}(1, n-r-1 ; n ; x)=(n-1) \int_{0}^{1}(1-t)^{n-2} \frac{\log (1-t x)}{(1-t x)^{n-r-1}} d t
$$

can be analytically continued from $x=0$ along each curve in $\mathbf{C}-\{1\}$. We conclude that

$$
z \mapsto E\left(r, r ; e^{z}, 1, \ldots, 1\right)=\exp \left(\frac{\frac{\partial}{\partial r}{ }_{2} F_{1}\left(1, n-r-1 ; n ; 1-e^{z}\right)}{{ }_{2} F_{1}\left(1, n-r-1 ; n ; 1-e^{z}\right)}\right)
$$

is analytic in $\mathbf{C}$, except for isolated essential singularities.

(4) In a similar (but much more direct) fashion we obtain that the function

$$
g_{u, v}(z)=\left[G\left(u, v ; e^{z}, 1, \ldots, 1\right)\right]^{u-v}=\frac{e^{u z}+n-1}{e^{v z}+n-1}
$$

has only simple poles and/or zeros, if $u \neq v$. Except for the case $n=2, u+v=0$ such poles and/or zeros actually exist. Taking limits we get

$$
G\left(u, u ; e^{z}, 1, \ldots, 1\right)=\exp \left(\frac{z}{1+(n-1) e^{-u z}}\right) .
$$


The function $z \mapsto G\left(u, u ; e^{z}, 1, \ldots, 1\right)$ is analytic in $\mathbf{C}$ with only essential isolated singularities, but without zeros. As before, we note that for $u \neq 0$ this set of essential singularities is not empty.

\section{Proof of the Theorem}

We first indicate sets of parameters for which $G$ and $E$ represent power means. For $n=2$ we have

$$
G\left(u, 0 ; x_{1}, x_{2}\right)=E\left(2 u, u ; x_{1}, x_{2}\right)=\left(\frac{x_{1}^{u}+x_{2}^{u}}{2}\right)^{1 / u} \quad(u \neq 0)
$$

and

$$
G\left(u,-u ; x_{1}, x_{2}\right)=E\left(r,-r ; x_{1}, x_{2}\right)=\left(x_{1} x_{2}\right)^{1 / 2},
$$

which implies that the power means belong to both, $G\left(u, v ; x_{1}, x_{2}\right)$ and $E\left(r, s ; x_{1}, x_{2}\right)$.

Let $n \geq 3$. Then we have

$$
\begin{aligned}
& G(1,0 ; \mathbf{x})=E(n, n-1 ; \mathbf{x})=\frac{1}{n} \sum_{k=1}^{n} x_{k}, \\
& G(0,0 ; \mathbf{x})=E(n-1,-1 ; \mathbf{x})=\prod_{k=1}^{n} x_{k}^{1 / n}
\end{aligned}
$$

and

$$
G(0,-1 ; \mathbf{x})=E(-1,-2 ; \mathbf{x})=\left(\frac{1}{n} \sum_{k=1}^{n} \frac{1}{x_{k}}\right)^{-1} .
$$

Thus, the arithmetic, geometric, and harmonic means are in the intersection of $G$ and $E$.

Now, we assume that $\mu$ is a mean which belongs to $G$ and $E$. Then there exist parameters $u, v, r$, and $s$ such that we have for all admissible $\mathbf{x}$ :

$$
\mu(\mathbf{x})=G(u, v ; \mathbf{x})=E(r, s ; \mathbf{x}) .
$$

If $u=v=0$, then $\mu$ is the geometric mean. Hence, let $(u, v) \neq(0,0)$. For the same reason, we exclude the case $n=2, u+v=0$. Further, we may suppose that

$$
u \geq v \text { and } r \geq s .
$$

Case 1. $u>v$ and $r>s$.

We set in (3.1): $x_{1}=e^{z}(z \in \mathbf{R}), x_{2}=\ldots=x_{n}=1$, and define $\alpha=$ $(u-v) /(r-s)$. This gives

$$
\left(f_{r, s}(z)\right)^{\alpha}=g_{u, v}(z),
$$

where $f_{r, s}$ and $g_{u, v}$ are as in (2.8) and (2.9), respectively. The relation (3.2) reveals that both functions can be extended meromorphically from $\mathbf{R}$ into $\mathbf{C}$. Since the function on the right-hand side is meromorphic with only simple poles and zeros (and, indeed, there are poles and/or zeros), we must have the same for the function on the left-hand side. According to Remark $(2), f_{r, s}$ has also only simple poles and zeros, so that $\alpha>0$ implies $\alpha=1$, or,

$$
s=r+v-u .
$$


Case 2. $u=v \neq 0$ or $r=s$.

We have

$$
E\left(r, s ; e^{z}, 1, \ldots, 1\right)=G\left(u, v ; e^{z}, 1, \ldots, 1\right) \quad(z \in \mathbf{R}) .
$$

The analytic continuation of both functions from $\mathbf{R}$ into $\mathbf{C}$ gives that (3.4) holds for all $z \in \mathbf{C}$, except for isolated singularities. If $r=s$, but $u \neq v$, then, according to Remarks (3) and (4), the function on the right-hand side of (3.4) has poles and/or zeros, while the function on the left-hand side can have only essential singularities, but no zeros. This cannot be true, and consequently, we have $u=v$, as well. A similar reasoning eliminates the possibility of $r \neq s$. Hence, (3.3) remains valid also in this case.

Our whole efforts so far were only to establish (3.3), which seems to be the key relation in the proof of the Theorem. We have not been able, not even with much more heavy use of computer algebra as in what follows, to ascertain the desired conclusion without making use of (3.3).

The final part of the proof is just calculation with the series expansion

$$
0 \equiv E\left(r, s ; e^{z}, 1, \ldots, 1\right)-G\left(u, v ; e^{z}, 1, \ldots, 1\right)=\sum_{k=0}^{\infty} p_{k}(r, s, u, v ; n) z^{k}
$$

Using (3.3) we get

$$
0=p_{2}(r, r+v-u, u, v ; n)=\frac{(n-1)[2(1+r-u)-n(1+u+v)]}{2 n^{2}(n+1)},
$$

which leads to

$$
r=\frac{(n+2) u+n v+n-2}{2} .
$$

Inserting (3.3) and (3.6) into (3.5), we obtain from $0=p_{3}=p_{4}$ the following two algebraic relations between $u, v, n$ :

$$
\begin{aligned}
0= & (n-2)\left[6 u v+n\left(u^{2}+4 u v+v^{2}-1\right)\right], \\
0= & 36 u v(u+v-1)+6 n\left[u^{3}+u^{2}(3 v-1)+u\left(3 v^{2}-3 v-1\right)+v^{3}-v^{2}-v+1\right] \\
& +n^{2}\left[-u^{3}+u^{2}(-28 v+1)+u\left(-28 v^{2}+10 v+1\right)-v^{3}+v^{2}+v-1\right] \\
& +n^{3}\left[-3 u^{3}+u^{2}(-9 v+1)+u\left(-9 v^{2}+4 v+3\right)-3 v^{3}+v^{2}+3 v-1\right] \\
& +n^{4}\left[u^{3}+4 u^{2} v+u\left(4 v^{2}-1\right)+v^{3}-v\right] .
\end{aligned}
$$

The Reduce command of Mathematica 4.0, when applied to this system, comes up with the complete set of solutions. Omitting those which are out of the range of our variables, we obtain:

If $n=2$, then either $u>0, v=0$, or $u=0, v<0$. And, if $n \geq 3$, then either $u=1, v=0$, or $u=0, v=-1$.

As seen in the first part of the proof, these choices correspond to the power means, if $n=2$, and to the arithmetic and harmonic means, if $n \geq 3$. This completes the proof of the Theorem.

\section{ACKNOWLEDGEMENT}

We thank the referee for helpful comments. 


\section{REFERENCES}

[1] G.E. Andrews, R. Askey, and R. Roy, Special Functions, Cambridge Univ. Press, Cambridge, 1999. MR 2000g:33001

[2] P.S. Bullen, D.S. Mitrinović, and P.M. Vasić, Means and Their Inequalities, Reidel, Dordrecht, 1988. MR 89d:26003

[3] D. Farnsworth and R. Orr, Gini means, Amer. Math. Monthly 93 (1986), 603-607. MR 88b:51031

[4] C. Gini, Di una formula comprensiva delle medie, Metron 13 (1938), 3-22.

[5] H.W. Gould and M.E. Mays, Series expansions of means, J. Math. Anal. Appl. 101 (1984), 611-621. MR 86a:26025

[6] G.H. Hardy, J.E. Littlewood, and G. Pólya, Inequalities, Cambridge Univ. Press, Cambridge, 1934.

[7] P. Kahlig and J. Matkowski, Functional equations involving the logarithmic mean, Z. Angew. Math. Mech. 76 (1996), 385-390. MR 97e:39017

[8] E.B. Leach and M.C. Sholander, Extended mean values, Amer. Math. Monthly 85 (1978), 84-90. MR 58:22428

[9] E.B. Leach and M.C. Sholander, Extended mean values II, J. Math. Anal. Appl. 92 (1983), 207-223. MR 85b:26007

[10] E.B. Leach and M.C. Sholander, Multi-variable extended mean values, J. Math. Anal. Appl. 104 (1984), 390-407. MR 86b:26033

[11] D.H. Lehmer, On the compounding of certain means, J. Math. Anal. Appl. 36 (1971), 183200. MR 43:7411

[12] L. Losonczi and Zs. Páles, Minkowski's inequality for two variable Gini means, Acta Sci. Math. (Szeged) 62 (1996), 413-425. MR 98c:26012

[13] L. Losonczi and Zs. Páles, Minkowski's inequality for two variable difference means, Proc. Amer. Math. Soc. 126 (1998), 779-789. MR 98e:26022

[14] Zs. Páles, Inequalities for sums of powers, J. Math. Anal. Appl. 131 (1988), 265-270. MR 89f:26024

[15] Zs. Páles, Inequalities for differences of powers, J. Math. Anal. Appl. 131 (1988), 271-281. MR 89f:26023

[16] Zs. Páles, Comparison of two variable homogeneous means, in: General Inequalities 6, Internat. Ser. Numer. Math., Vol. 103, Birkhäuser, Basel, 1992, 59-70. MR 94b:26016

[17] A.O. Pittenger, The logarithmic mean in $n$ variables, Amer. Math. Monthly 92 (1985), 99104. MR 86h:26012

[18] G. Pólya and G. Szegö, Isoperimetric Inequalities in Mathematical Physics, Princeton Univ. Press, Princeton, 1951. MR 13:270d

[19] K.B. Stolarsky, Generalizations of the logarithmic mean, Math. Mag. 48 (1975), 87-92. MR 50:10186

Morsbacher Str. 10, 51545 Waldbröl, Germany

E-mail address: alzer@wmax03.mathematik.uni-wuerzburg.de

Department of Mathematics, University of Würzburg, Am Hubland, 97074 Würzburg, GERMANY

E-mail address: ruscheweyh@mathematik.uni-wuerzburg.de 